\title{
NON-MODAL ANALYSIS OF MULTIGRID SCHEMES FOR THE HIGH-ORDER FLUX RECONSTRUCTION METHOD
}

\section{AURELIO HURTADO-DE-MENDOZA ${ }^{1,2, *}$, JIAQING KOU ${ }^{1,2}$, SAUMITRA JOSHI ${ }^{1,2}$, KUNAL PURI ${ }^{1}$, CHARLES HIRSCH ${ }^{1}$ AND ESTEBAN FERRER ${ }^{2}$}

\author{
${ }^{1}$ NUMECA International S.A. \\ Chaussée de la Hulpe 187, Brussels B-1170 Belgium \\ *aurelio.hurtadodemendoza@numeca.be \\ ${ }^{2}$ ETSIAE-UPM School of Aeronautics, Universidad Politécnica de Madrid \\ Plaza Cardenal Cisneros 3, Madrid E-28040 Spain \\ E-mail address and URL
}

Key words: High-order, Flux Reconstruction, convergence acceleration, multigrid, non-modal analysis, stability.

\begin{abstract}
The present study introduces an application of the non-modal analysis to multigrid operators with explicit Runge-Kutta smoothers in the context of Flux Reconstruction discretizations of the linear convection-diffusion equation. A dissipation curve is obtained that reflects upon the convergence properties of the multigrid operator. The number of smoothing steps, the type of cycle (V/W) and the combination of $p$ - and $h$-multigrid are taken into account in order to find those configurations which yield faster convergence rates. The analysis is carried out for polynomial orders up to $P=6$, in $1 \mathrm{D}$ and 2D for varying degrees of convection (Péclet number), as well as for high aspect ratio cells. The non-modal analysis can support existing evidence on the behaviour of multigrid schemes. W-cycles, a higher number of coarse-level sweeps or the combined use of $h p$-multigrid are shown to increase the error dissipation, while higher degrees of convection and/or high aspect-ratio cells both decrease the error dissipation rate.
\end{abstract}

\section{INTRODUCTION}

For industrial Computational Fluid Dynamics (CFD), multigrid methods and convergence acceleration is critical for quick turnaround times. It is well established that high-order methods like DiscontinuousGalerkin (DG, [1]) and Flux Reconstruction (FR, [2]) offer significant advantages over traditional, secondorder Finite Volume methods (FVM). They are more efficient for a given level of accuracy [3], can effectively utilize modern computer architectures like GPUs and are quasi mesh-independent. A drawback is that these methods are less mature and robust than their FVM counterparts. This has been the motivating factor in studying the numerical properties and stability limits of such schemes for under-resolved simulations of turbulent flows $[4,5]$.

The Flux Reconstruction (FR) method, recently devised by Huynh $[2,6]$ to solve conservation laws, is formulated as a unifying approach for discontinuous Finite Element methods such as DG and Spectral Difference (SD). The method has been further extended by Vincent, Castonguay and Jameson [7, 
8] through the family of Energy-Stable Flux Reconstruction (ESFR) schemes, and it has revealed itself particularly suited for the simulation of turbulent flows using implicit Large Eddy Simulations [4, 5, $9,10]$. Several studies about the numerical properties of FR by means of von Neumann analysis exist in the literature $[11,12,13,14]$, mainly in the context of linear advection and advection-diffusion. Despite good experimental agreement, the von Neumann analysis has an inherent disadvantage when applied to high-order methods, since more than one eigenmode per element is obtained which forces a distinction between the primary (physical) eigenmode and the spurious ones, which are disregarded altogether. Nevertheless, near the high frequency region all eigenmodes have comparable influence in the numerical behaviour, leading Alhawwary and Wang [15] to propose the combined-mode analysis [16], which extracts a global dispersion/dissipation based on the relative energy of the eigenmodes. Similarly, Fernández et al. [17] have also introduced the non-modal analysis to assess the dissipation properties without differentiating between physical or spurious eingemodes, providing insights into nonlinear instabilities in under-resolved flow simulations at high polynomial orders.

Literature related to the analysis of these methods for convergence acceleration via multigrid remains insufficient. Multigrid for $p$-type Finite Element methods, often referred to as $p$-multigrid, was initially developed in $[18,19,20]$ and has mostly targeted the simulation of fluid flows using DG $[21,22,23$, $24,25,26,27]$. In the so-called $p$-multigrid, lower polynomial orders act as coarser levels, following a similar idea to the conventional $h$-multigrid, while the simultaneous use of the two is known as $h p$ multigrid [28]. In the context of multigrid for conventional low-order methods, numerous applications of the von Neumann analysis to two-level schemes exist in the literature $[29,30,31]$, while of the analysis of $p$-and $h p$-multigrid the influence of the mullig tively less attention. Fu high aspect-ratio meshes, explored.

The objective of this stuct
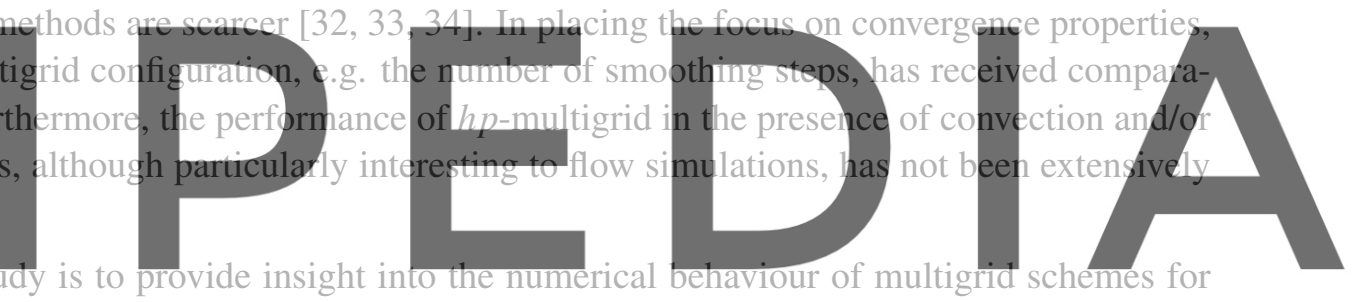

high-order methods, where the need for robust and efficient algorithms is especially relevant. By using

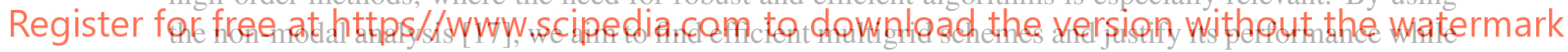
retaining its applicability to a wide range of problems, from pure diffusion to high-Péclet convection.

\section{FLUX RECONSTRUCTION DISCRETIZATION OF THE LINEAR CONVECTION- DIFFUSION EQUATION}

The linear convection-diffusion equation with constant coefficients $\boldsymbol{a}$ and $\boldsymbol{v} \geq 0$ for a scalar $u(\boldsymbol{x}, t)$ reads:

$$
\frac{\partial u}{\partial t}+\nabla \cdot\left(\boldsymbol{F}^{\mathrm{cnv}}+\boldsymbol{F}^{\mathrm{diff}}\right)=0, \quad \boldsymbol{F}^{\mathrm{cnv}}=\boldsymbol{a} u, \quad \boldsymbol{F}^{\mathrm{diff}}=-v \nabla u .
$$

This section outlines the discretization of this equation in 1D by means of the Flux Reconstruction method. For more details, the reader is referred to the literature $[2,6]$.

The computational domain is divided in cells $C_{j}$, with center $x_{j}$ and width $h_{j}$. A cell-local reference space is parametrized by coordinate $-1 \leq \xi \leq 1$ and the mapping from physical space to reference space is as follows:

$$
\xi(x)=\frac{2}{h_{j}}\left(x-x_{j}\right), \frac{\mathrm{d} \xi}{\mathrm{d} x}=\frac{2}{h_{j}}, \forall x \in C_{j} .
$$


Inside cell $C_{j}$, the solution is approximated at some quadrature points $\xi_{k}$ (in this study, Gauss-Legendre), with $k=0, \ldots, P$, locally defining a polynomial of order $P$ which can be represented by a vector of nodal values $\boldsymbol{u}_{j}$. The spatial discretisation using the FR method consists of seven steps:

1. Interpolation of the solution at the cell interface points, given by $\xi= \pm 1$, to obtain $u_{j}^{L}$ and $u_{j}^{R}$.

2. Computation of the common solution values at the interface points. Here we use the Local Discontinuous Galerkin (LDG) approach [35] with $\beta=0, \tau=1$ :

$$
u_{j}^{I, L}=\frac{u_{j-1}^{R}+u_{j}^{L}}{2}-\beta\left(u_{j-1}^{R}-u_{j}^{L}\right) \quad, \quad u_{j}^{I, R}=\frac{u_{j}^{R}+u_{j+1}^{L}}{2}-\beta\left(u_{j}^{R}-u_{j+1}^{L}\right) .
$$

3. Computation of the gradient of the solution, $\boldsymbol{q}_{j}$, by summing up the discontinuous gradient, which results from directly evaluating the derivative of $\boldsymbol{u}_{j}$, and the correction term, proportional both to the jumps in the solution at the interfaces and to the so-called correction functions - see $[36,6]$ for more details. As is done in this study, the use of Radau polynomials as correction functions yields a scheme which is numerically equivalent to a nodal DG method.

$$
\boldsymbol{q}_{j}=D_{j} \boldsymbol{u}_{j}+\left(u_{j}^{I, L}-u_{j}^{L}\right) \boldsymbol{g}_{j}^{\prime L}+\left(u_{j}^{I, R}-u_{j}^{R}\right) \boldsymbol{g}_{j}^{\prime R}
$$

4. Interpolation of the fluxes at the interface points (e.g. $F_{j}^{\mathrm{cnv}, L}$ and $F_{j}^{\mathrm{diff}, L}$ for the left boundary).
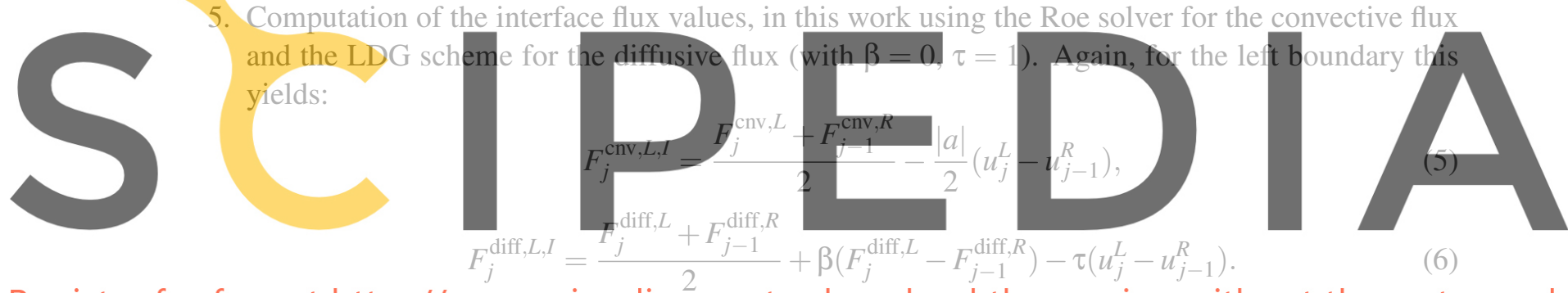

(6)

Register for free at https//www.scipedia.com to download the version without the watermark

6. Divergence of the corrected fluxes, in a similar way to step 3:

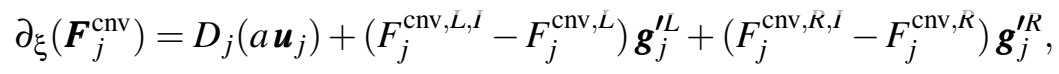

$$
\begin{aligned}
& \partial_{\xi}\left(\boldsymbol{F}_{j}^{\mathrm{diff}}\right)=D_{j}\left(-v \boldsymbol{\boldsymbol { q } _ { j }}\right)+\left(F_{j}^{\mathrm{diff}, L, I}-F_{j}^{\mathrm{diff}, L}\right) \boldsymbol{g}_{j}^{L}+\left(F_{j}^{\mathrm{diff}, R, I}-F_{j}^{\mathrm{diff}, R}\right) \boldsymbol{g}_{j}^{\prime R} .
\end{aligned}
$$

7. Transformation from reference space to physical space and evaluation of the time derivative:

$$
\frac{\mathrm{d} \boldsymbol{u}_{j}}{\mathrm{~d} t}=-\frac{\mathrm{d} \xi}{\mathrm{d} x} \frac{\partial}{\partial \xi}\left(\boldsymbol{F}_{j}^{\mathrm{cnv}}+\boldsymbol{F}_{j}^{\mathrm{diff}}\right)
$$

This completes the spatial discretization. The above can be rewritten in a compact matrix form:

$$
\frac{\mathrm{d} \boldsymbol{u}_{j}}{\mathrm{~d} t}=\sum_{i=j-2}^{j+2} \mathcal{A}_{j, i} \boldsymbol{u}_{i},
$$


where $\mathcal{A}_{j, i}$ is an operator defined in element $C_{j}$ acting on neighbouring solution vectors $\boldsymbol{u}_{i}$. By using some shift matrices $\mathcal{T}_{i, j}$ such that $\boldsymbol{u}_{i}=\mathcal{T}_{i, j} \boldsymbol{u}_{j}$, whose form depends primarily upon the initial condition, we obtain a fully local form of the spatial discretization:

$$
\frac{\mathrm{d} \boldsymbol{u}_{j}}{\mathrm{~d} t}(t)=\mathcal{A}_{j} \boldsymbol{u}_{j}(t)=\left(\sum_{i=j-2}^{j+2} \mathcal{A}_{j, i} \mathcal{T}_{i, j}\right) \boldsymbol{u}_{j}(t) .
$$

For time-marching we will use an explicit Runge-Kutta polynomial $\mathcal{P}_{S}^{N}(x)$ with $S$ stages that is $N$-th order accurate in time. For a given time-step $\Delta t=t_{n+1}-t_{n}$ the fully discrete operator yields:

$$
Z=\mathcal{P}_{S}^{N}(\Delta t \mathcal{A}) \quad, \quad \boldsymbol{u}\left(t_{n+1}\right)=Z \boldsymbol{u}\left(t_{n}\right),
$$

\section{ANALYSIS OF MULTIGRID OPERATORS}

Considering the following linear system:

$$
\mathcal{A} u=f,
$$

an inexact guess of the solution will produce a residual $\boldsymbol{r}=\boldsymbol{f}-\mathcal{A} \boldsymbol{u}$, which can be quickly damped using the multigrid method as it efficiently eliminates low wavenumber errors. The iterative process can be written - in the case of a zero-right-hand side in equation 13 - as follows:
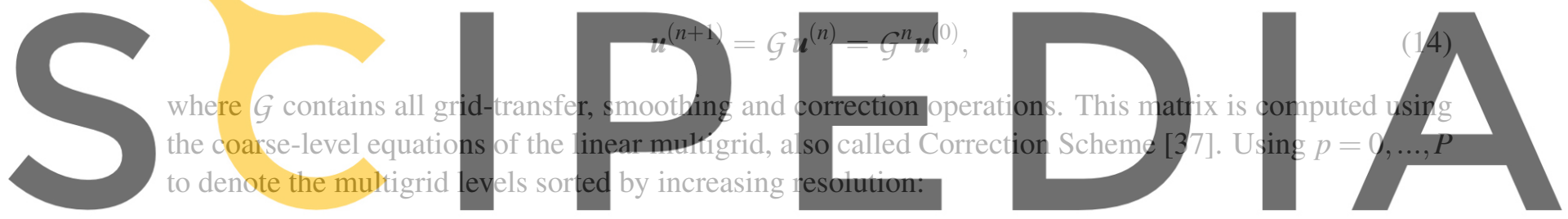

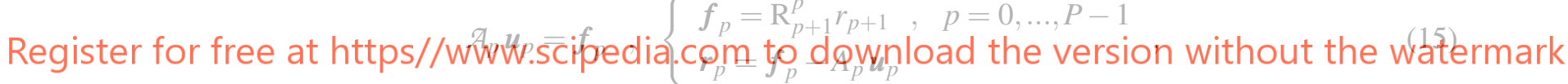

where $\mathbf{R}_{p+1}^{p}$ is the restriction matrix from level $p+1$ to level $p$. Starting from an initial guess $\left(\boldsymbol{u}_{P}\right)_{0}^{\mathrm{do}}$, the fine-level pre-smoothing yields:

$$
\left(\boldsymbol{u}_{P}\right)_{m_{P}}^{\mathrm{do}}=Z_{m, P}\left(\boldsymbol{u}_{P}\right)_{0}^{\mathrm{do}}+\tilde{Z}_{m, P} \boldsymbol{f}_{P}
$$

$m_{p}$ is the number of smoothing steps (sweeps) at level $p$, and superscripts "do" and "up" denote the solutions obtained in the down-cycle and the up-cycle. For an explicit marching with a Runge-Kutta method, the smoothing operators $Z_{m, p}$ and $\tilde{Z}_{m, p}$ are:

$$
Z_{m, p}=\left(Z_{p}\right)^{m_{p}} \quad, \quad \tilde{Z}_{m, p}=\Delta t\left(\sum_{n=0}^{m_{p}-1}\left(Z_{p}\right)^{n}\right) \tilde{\mathcal{P}}\left(\Delta t \mathcal{A}_{p}\right),
$$

where $\tilde{\mathcal{P}}(x)=\left(1-\mathcal{P}_{S}^{N}(x)\right) / x$ is obtained from the Runge-Kutta polynomial. After some algebra, we obtain that the solutions in the down-cycle at the coarse levels are: 


$$
\left(\boldsymbol{u}_{j}\right)_{m_{p}}^{\mathrm{do}}=\tilde{Z}_{m, p}\left[\prod_{k=p+1}^{P-1} \mathrm{R}_{k}^{k-1}\left(I_{k}-\mathcal{A}_{k} \tilde{Z}_{m, k}\right)\right] \mathrm{R}_{P}^{P-1} \boldsymbol{r}_{P}, \quad p=P-1, \ldots, 0
$$

with $I_{p}$ the $(p+1) \times(p+1)$ identity matrix. The up-cycle consists two steps, prolongation and smoothing:

$$
\begin{aligned}
& \left(\boldsymbol{u}_{p}\right)_{0}^{\text {up }}=\left(\boldsymbol{u}_{p}\right)_{m_{p}}^{\mathrm{do}}+\mathbf{R}_{p-1}^{p}\left(\boldsymbol{u}_{p-1}\right)_{m_{p-1}}^{\text {up }} \\
& \left(\boldsymbol{u}_{p}\right)_{m_{p}}^{\text {up }}=Z_{m, p}\left(\boldsymbol{u}_{p}\right)_{0}^{\mathrm{up}}+\tilde{Z}_{m, p} \boldsymbol{f}_{p}
\end{aligned}
$$

Hence, the up-cycle solutions are expressed in terms of the down-cycle solutions according to:

$$
\left(u_{p}\right)_{m_{p}}^{\mathrm{up}}=\left(\prod_{l=0}^{p-1} z_{m, p-l} \mathrm{R}_{p-l-1}^{p-l}\right)\left(u_{0}\right)_{m_{0}}^{\mathrm{do}}+\sum_{k=1}^{p}\left[\left(\prod_{l=0}^{p-k-1} z_{m, p-l} \mathrm{R}_{p-l-1}^{p-l}\right)\left(z_{m, k}+I_{k}\right)\left(u_{k}\right)_{m_{k}}^{\mathrm{do}}\right] \text {. }
$$

Combining equations 20 and 18 we can express the solution vector $\left(\boldsymbol{u}_{P}\right)_{m_{P}}^{\mathrm{up}}$ in terms of the initial guess $\left(\boldsymbol{u}_{P}\right)_{0}^{\mathrm{do}}$ and $\boldsymbol{f}_{P}$. This allows to express the multigrid operator as a single matrix for whichever polynomial order and sweep pattern. The inclusion of $h$-levels in addition to $p$-multigrid levels is straightforward; so is the computation of a W-cycle matrix by including coarse-grid revisits.
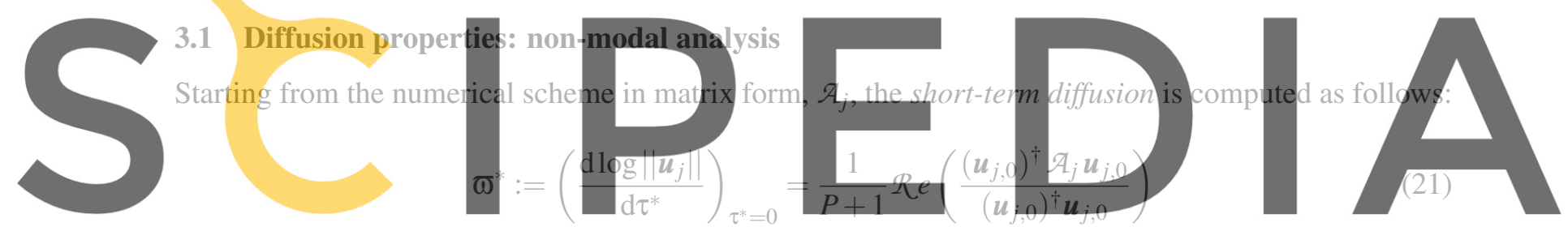

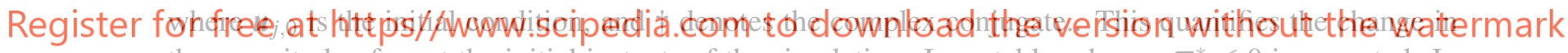
the magnitude of $u_{j}$ at the initial instants of the simulation. In a stable scheme, $\omega^{*} \leq 0$ is expected. In order to obtain an analogue curve in the case of a fully-discrete scheme, one may take the following approximation:

$$
\varpi^{*}:=\left(\frac{\mathrm{d} \log \left\|\boldsymbol{u}_{j}\right\|}{\mathrm{d} \tau^{*}}\right)_{\tau^{*}=0} \simeq \frac{1}{P+1} \mathcal{R} e\left(\frac{1}{\Delta t} \log \left(\frac{\left(\boldsymbol{u}_{j, 0}\right)^{\dagger} \boldsymbol{Z}_{j} \boldsymbol{u}_{j, 0}}{\left(\boldsymbol{u}_{j, 0}\right)^{\dagger} \boldsymbol{u}_{j, 0}}\right)\right)
$$

which is consistent with the original equation in the time-continuous limit $\Delta t \rightarrow 0$. By using $\mathcal{G}_{j}$ instead of $Z_{j}$, we are able to analyse a multigrid operator using an explicit expression which depends on the scheme's configuration. Similar to a von Neumann analysis, the dispersion curve is symmetric; however, it is not periodic. Finally, as we are interested in solving the steady-state problem $\mathcal{A}_{j} \boldsymbol{u}_{j}=0$, for a given multigrid operator we may relate a higher short-term diffusion (more negative values of $\varpi^{*}$ ) with a faster convergence rate. 


\section{RESULTS OF THE ANALYSIS}

We begin by focusing in multigrid operators for the $1 \mathrm{D}$ diffusion equation with $v=1$. In a similar way to the von Neumann dissipation curves, the non-modal dissipation curves show the damping factor, $\varpi^{*}(\phi)$, as a function of the frequency of the initial wave. This frequency is expressed in terms of a normalized wavenumber, $\phi=\kappa h /(P+1)$.

\subsection{VON NEUMANN ANALYSIS AND NON-MODAL ANALYSIS}

In figure 1, we perform a comparison between the non-modal dissipation curve and the dissipation plots obtained from a von Neumann analysis, where the latter are extracted from the eigenvalues of the multigrid operator. Loosely speaking, each eigenmode is representative of the behaviour of one multigrid level, and dominates over the others in its corresponding region of the frequency spectrum. Thus, the damping in the low frequency spectrum is handled by the coarsest level, and the same happens for the finest level in the high-wavenumber range. It can clearly be seen in figure 1 how the non-modal curve approaches that of the relevant eigenmode at the corresponding intervals.
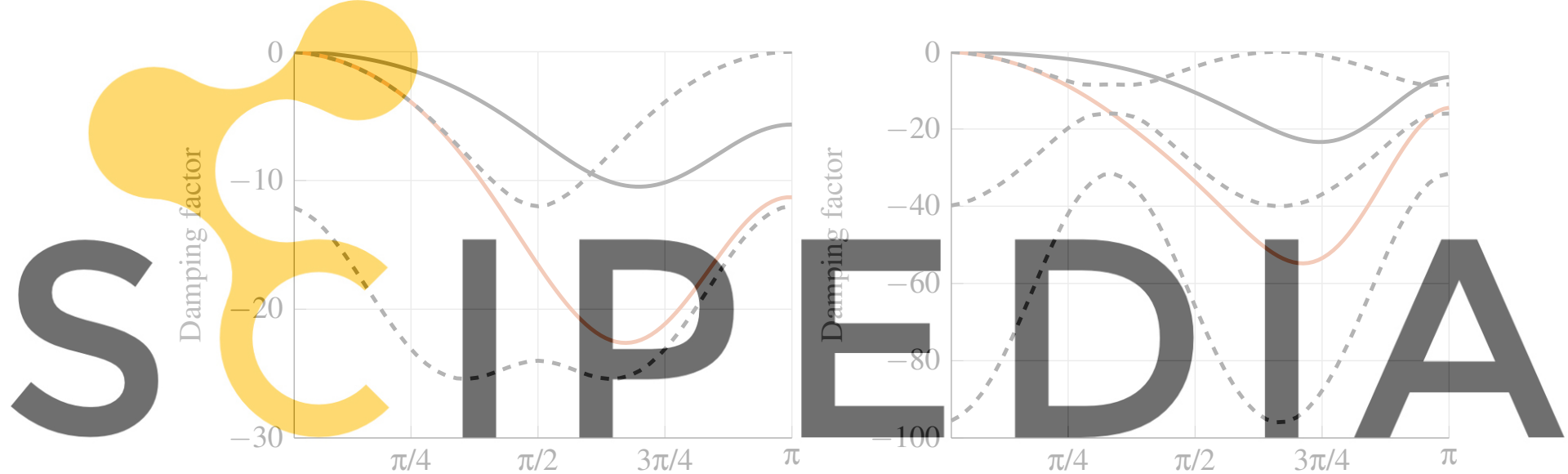

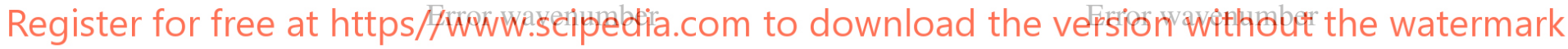
(a) $P=1$

(b) $P=2$

Figure 1: For a V-cycle p-multigrid operator in 1D diffusion, we plot: non-modal dissipation curve (red), eigenmodes (dashed lines) and non-modal dissipation of explicit Runge-Kutta marching (no-multigrid, black line) at orders $P=1,2$, in the time-continuous limit.

\subsection{EFFECT OF THE MULTIGRID CONFIGURATION}

In order to isolate the effect of each multigrid parameter on its own, we begin by studying the simplest configurations in 1D diffusion and progressively change the sweep pattern, the type of cycle, and add the combination of $h p$-multigrid. In figure 2, V-cycle $p$-multigrid is considered with only one smoothing step at each level. The dissipation is increased consistently with the polynomial order in the whole wavenumber range, suggesting that the multigrid effectiveness would increase with the polynomial order, since so does the number of multigrid levels.

Next, we increase the number of coarse-level sweeps within the same V-cycle configuration, following 


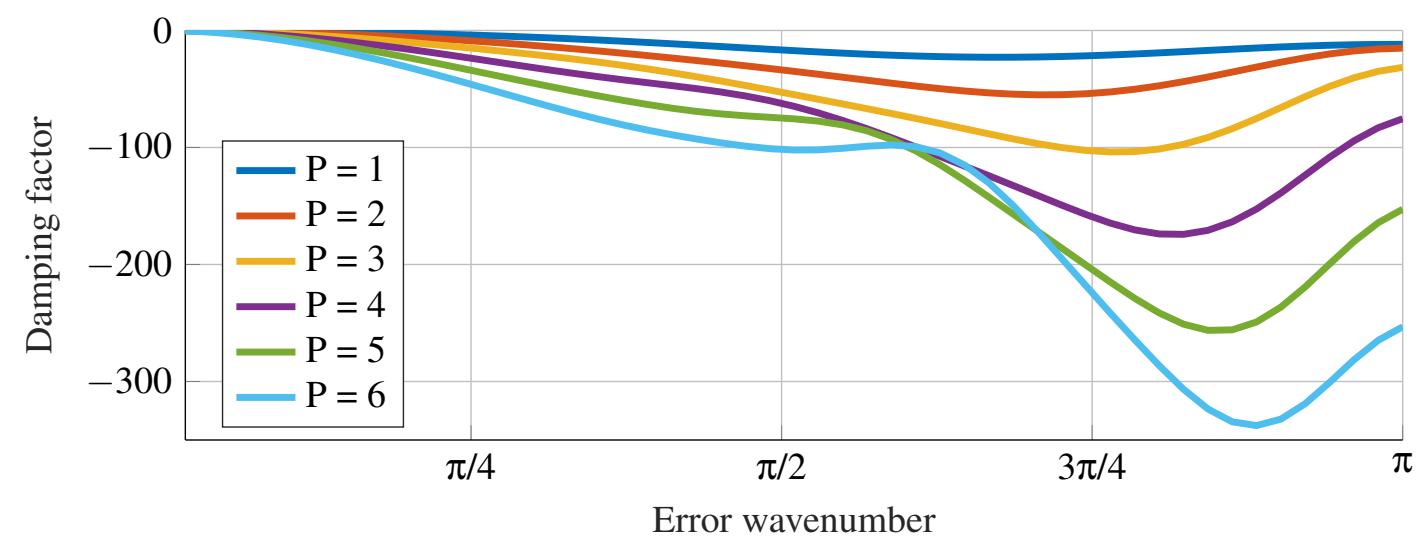

Figure 2: Non-modal dissipation curves of the V-cycle $p$-multigrid operator at polynomial orders $P=$ $1, \ldots, 6$, in the time-continuous limit.

usual practice given that coarse-level smoothing is relatively inexpensive. In addition to the constant, allones sweep pattern (SP), we consider a linear sweep pattern (the number of smoothing steps increases linearly with the level numer) and a geometric sweep pattern (the number of smoothing steps increases geometrically). The result can be seen in figure 3, confirming the effectiveness of increasing coarse-level smoothing not only in the low wavenumber range, but also in the rest of the frequency spectrum.
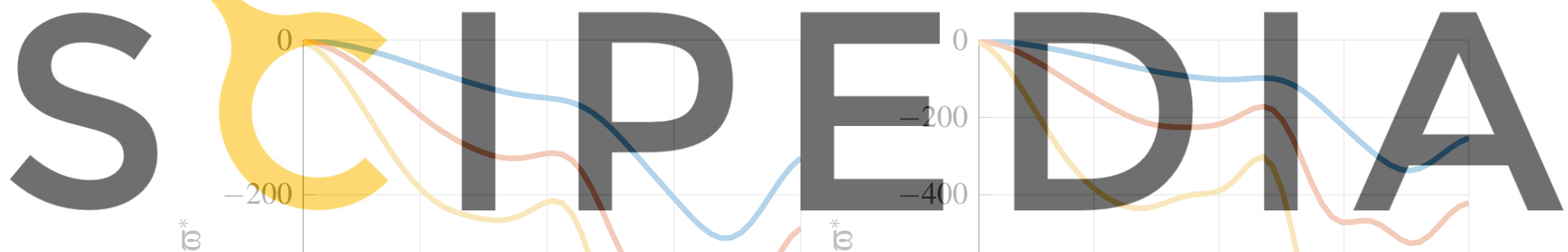

Register for free at https//www.scipedia.com to c

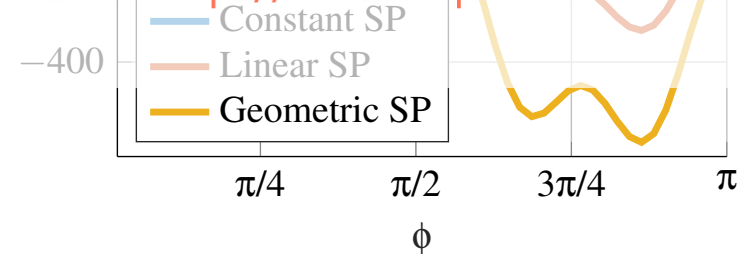

(a) $P=5$

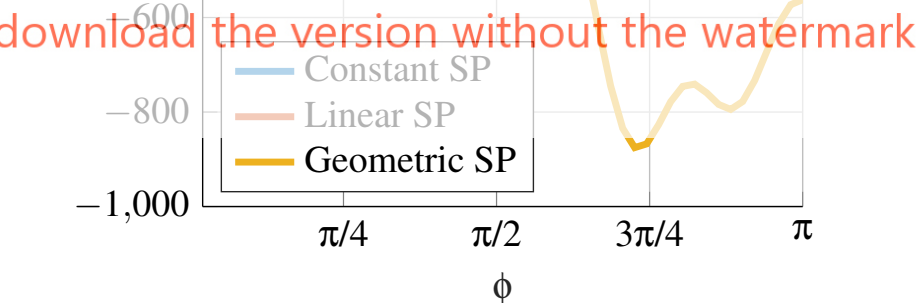

(b) $P=6$

Figure 3: Dissipation curves of V-cycle $p$-multigrid operators at $P=5,6$ with different sweep patterns.

Keeping the geometric sweep pattern, figure 4(a) compares the dissipation rate at $P=2$ of a V-cycle operator against that of $\mathrm{W}$-cycle operators with an increasing number of $\mathrm{w}$-sweeps. Two $\mathrm{w}$-sweeps means are equivalent to 2 embedded V-cycles between $p=1$ and $p=0$. While the fine-level smoothing steps are the same, the W-cycles add a significant amount of low- and mid-frequency dissipation. A complementary strategy consist in adding additional coarser meshes in $h$ to the overall multigrid algorithm, which results 
into the combined $h p$-multigrid operator depicted in figure 4(b). While most of the frequecy spectrum remains unchanged, the changes in the slope of the dissipation curve in the zero-frequency limit can translate into significant improvements in the convergence rate.

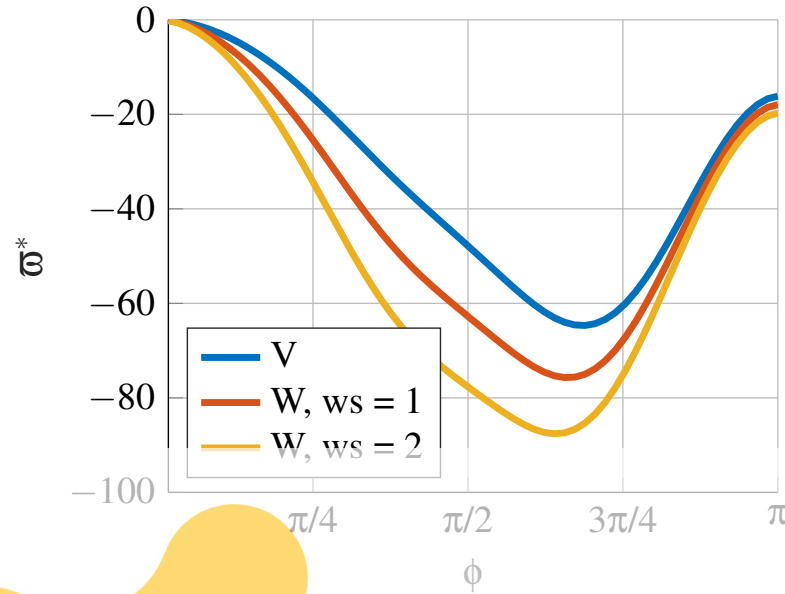

(a) Dissipation curves of V-cycle and W-cycle $p$-multigrid(b) Dissipation curves of V-cycle $p$-multigrid and $h p$ -

operators. "ws" stands for the number of w-sweeps. multigrid operators.

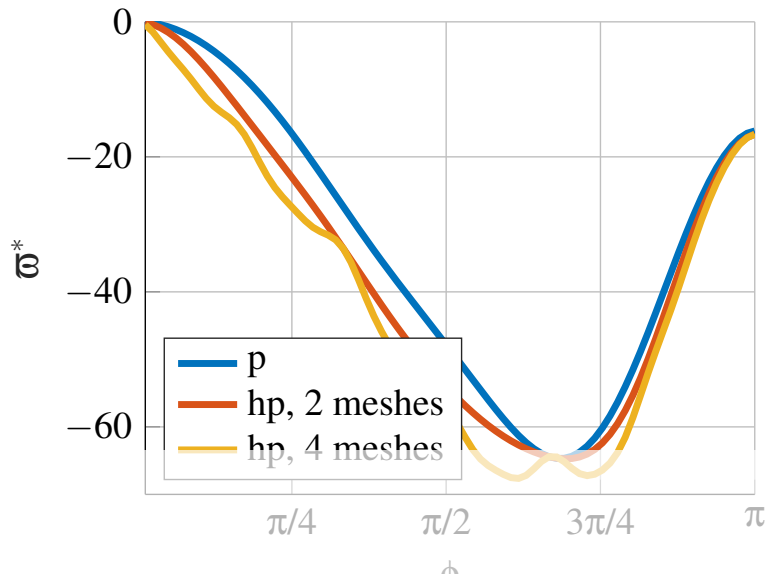

\section{Figure 4: Dissipation curves of several multigrid operators at $P=2$.}
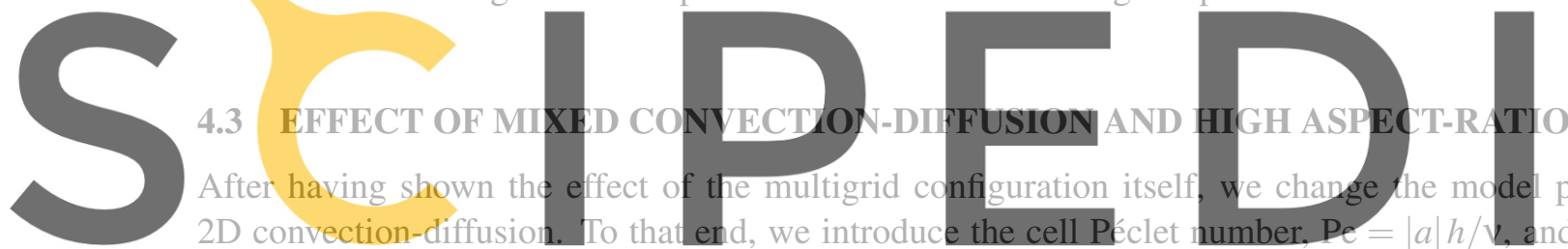

2D convection-diffusion. To that end, we introduce the cell Péclet

Recall that in 2D, two independent

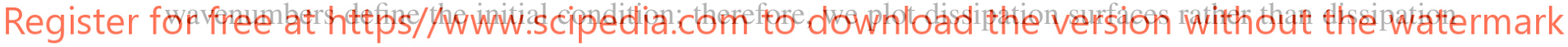
curves.

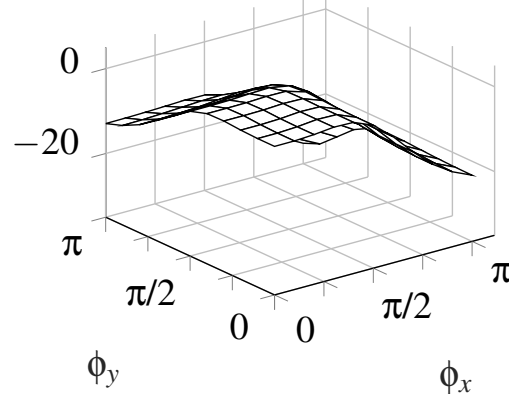

(a) $r_{h}=1$

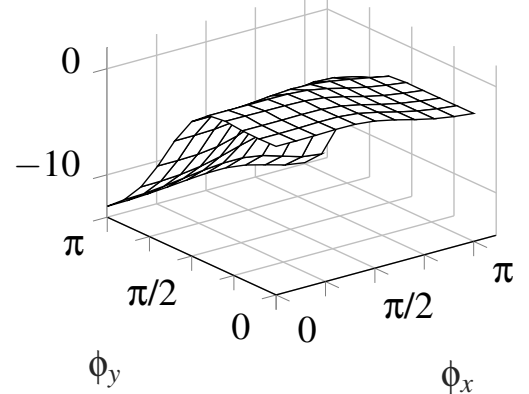

(b) $r_{h}=10$

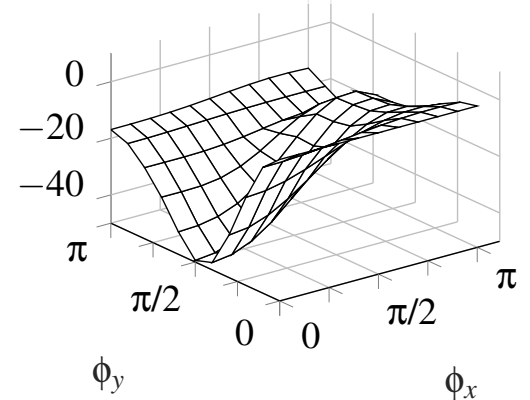

(c) $r_{h}=1000$

Figure 5: Scaled non-modal dissipation $\varpi^{*} r_{h}^{-1}$ of linear convection-diffusion at $\mathrm{Pe}=10^{3}$ with V-cycle $p$-multigrid at increasing aspect-ratios. 
Figure 5 plots the scaled non-modal dissipation of V-cycle $p$-multigrid operators at increasing aspect ratios, where the scaled damping factor $\varpi^{*} r_{h}^{-1}$ accounts for the decrease in the time-step limit as the aspect-ratio increases. $\mathrm{Pe}=10^{3}$ implies $v=0.001$, and we choose $a_{x}=1$ and $a_{y}=0$, so convection happens along the $x$-axis. In the case of a square cell (aspect-ratio 1), the curve is smooth and symmetric. This situation changes drastically as the aspect-ratio is increased; as a result, the $y$-component of lowfrequency component seems to remain largely undamped.

In order to increase the dissipation in high aspect-ratio meshes, one may propose to use $\mathrm{W}$-cycles and $h p$-multigrid. The resulting behaviour is shown in figure 6 . In line with the conclusions drawn from the 1D dissipation curves, the use of these multigrid operators results into an increased dissipation in the overall spectrum.

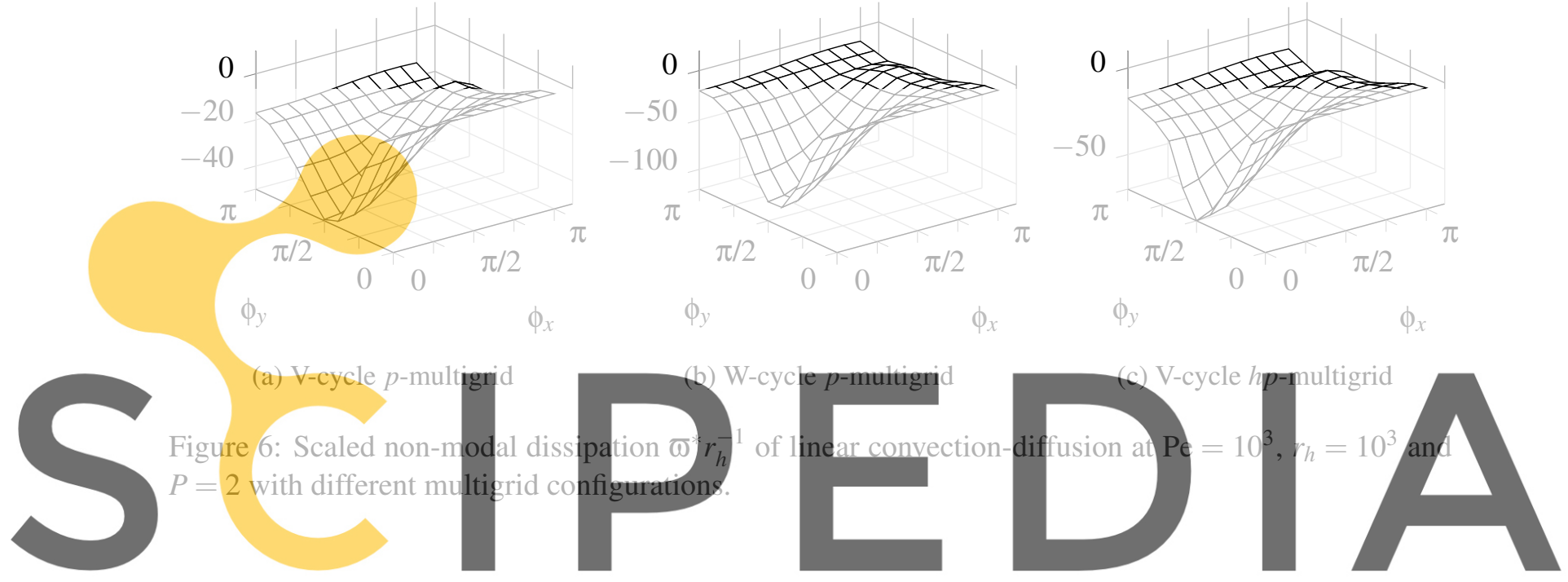

5 CONCLUSIONS

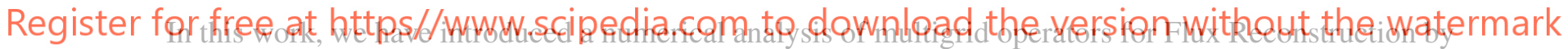

means of the non-modal analysis. The analysis has been carried out in the context of 1D and 2D linear

convection-diffusion, and the focus has been on the effect of the multigrid parameters and the presence of convection-diffusion and high aspect-ratios meshes. The non-modal dissipation is shown to increase with the polynomial order. Low-wavenumber dissipation could be increased by either raising the number of coarse-level sweeps (by using linear or geometric sweep patterns) or by using W-cycle multigrid instead of V-cycle. Likewise, the combined use of $h p$-multigrid resulted into an increase of the dissipation in the limit of zero-frequency.

In $2 \mathrm{D}$, the non-modal dissipation was shown to be significantly affected by the presence of dominant convection and high aspect-ratios. Although a decrease in the multigrid efficiency shown for certain regions of the frequency spectrum, the conclusions drawn from the 1D dissipation curves reflect upon theoretical improvements in convergence for the $2 \mathrm{D}$ cases considered. 


\section{ACKNOWLEDGEMENTS}

This project has received funding from the European Union's Horizon 2020 research and innovation programme under the Marie Skłodowska-Curie grant agreement MSCA ITN-EID-GA ASIMIA No 813605.

\section{References}

[1] W. H. Reed and T. R. Hill. Triangular mesh methods for the neutron transport equation. Tech. rep. LA-UR-73-479. Los Alamos National Laboratory, 1973.

[2] H. T. Huynh. "A Flux Reconstruction Approach to High-Order Schemes Including Discontinuous Galerkin Methods”. In: AIAA 18th Computational Fluid Dynamics Conference. 2007. DOI: 10 . 2514/6.2007-4079.

[3] Z. J. Wang et al. "High-order CFD methods: current status and perspective". In: Int. J. Numer. Meth. Fluids (2013). DOI: 10.1002/fld.3767.

[4] Jonathan R. Bull and Antony Jameson. "High-Order Flux Reconstruction Schemes for LES on Tetrahedral Meshes". In: Progress in Hybrid RANS-LES Modelling. Springer International Publishing, 2015, pp. 69-79. DOI: 10.1007/978-3-319-15141-0_5.

[5] N.A. Loppi et al. "A high-order cross-platform incompressible Navier-Stokes solver via artificial compressibility with application to a turbulent jet". In: Computer Physics Communications 233 (2018), pp. 193-205. DOI: 10.1016/j.cpc.2018.06.016.

[6] H. T. Huynh. "A Reconstruction Approach to High-Order Schemes Including Discontinuous Galerkin for Diffusion". In: AIAA 47th Aerospace Sciences Meeting. 2009. D OI: 10.2514/6.2009-403.

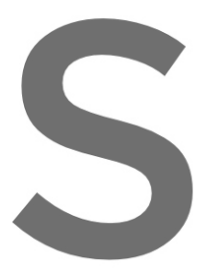

[7] P. E. Vincent, P, Reconstruction S $9420-z$.

[8] P. Castonguay, $\mathrm{P}$ Reconstruction DOI: $10.1007 / \mathrm{s}: 00$
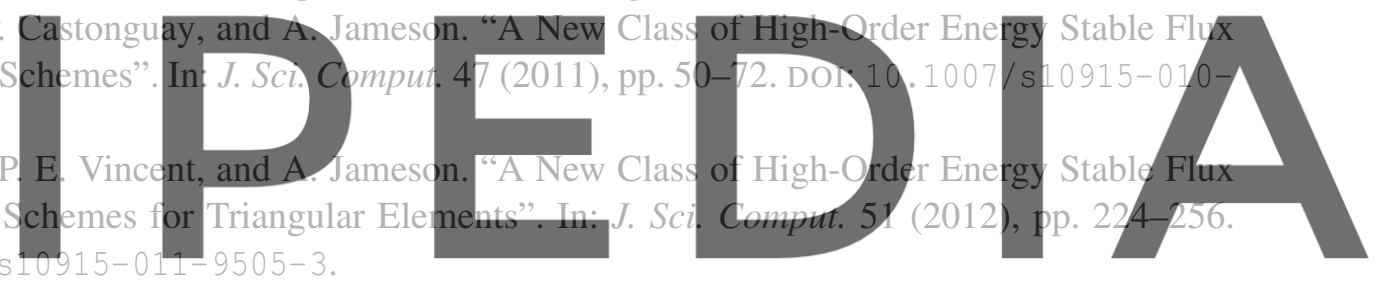

[9] N. A. Loppi et al. "Locally adaptive pseudo-time stepping for high-order Flux Reconstruction".

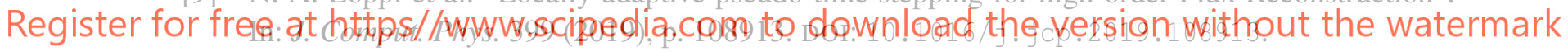

[10] Mohammad A. Alhawwary and Zhi J. Wang. "DNS and LES of the flow over the T106C turbine using the high-order FR/CPR method". In: AIAA Scitech 2020 Forum. 2020. DOI: $10.2514 / 6$. $2020-1572$.

[11] P. E. Vincent, P. Castonguay, and A. Jameson. "Insights from von Neumann analysis of high-order flux reconstruction schemes”. In: J. Comput. Phys. 230 (2011), pp. 8134-8154. DOI: 10.1016/ j. jcp.2011.07.013.

[12] Jerry Watkins, Kartikey Asthana, and Antony Jameson. "A numerical analysis of the nodal Discontinuous Galerkin scheme via Flux Reconstruction for the advection-diffusion equation". In: Computers \& Fluids 139 (2016), pp. 233-247. DOI: 10.1016/ j.compfluid.2016.09.013.

[13] B. C. Vermeire and P. E. Vincent. "On the behaviour of fully-discrete flux reconstruction schemes". In: Comput. Methods Appl. Mech. Engrg. 315 (2017), pp. 1053-1079. DOI: 10 . 1016 / j . cma . 2016.11.019.

[14] Kartikey Asthana, Jerry Watkins, and Antony Jameson. "On consistency and rate of convergence of Flux Reconstruction for time-dependent problems". In: Journal of Computational Physics 334 (2017), pp. 367-391. DOI: 10.1016/j.jcp.2017.01.008. 
[15] M. Alhawwary and Z. J. Wang. "Fourier analysis and evaluation of DF, FD and compact difference methods for conservation laws." In: J. Comput. Phys. 373 (2018), pp. 835-862. DOI: 10.1016/ j . jcp.2018.07.018.

[16] Mohammad A. Alhawwary and Zhi J. Wang. "A study of DG methods for diffusion using the combined-mode analysis". In: AIAA Scitech 2019 Forum. 2019. DOI: 10.2514/6.2019-1157.

[17] P. Fernandez et al. "Non-modal analysis of spectral element methods: Towards accurate and robust large eddy simulations". In: J. Comput. Methods Appl. Mech. Engrg. 346 (2019), pp. 43-62. DOI: 10.1016/j.cma.2018.11.027.

[18] E.M. Rønquist and A.T. Patera. "Spectral element multigrid. I. Formulation and numerical results". In: Journal of Scientific Computing 2.4 (1987), pp. 389-406. DOI: 10.1007/bf01061297.

[19] David Gottlieb and Steven A. Orszag. Numerical Analysis of Spectral Methods. Society for Industrial and Applied Mathematics, 1977. DOI: 10 .1137/1.9781611970425. eprint: https: //epubs.siam.org/doi/pdf/10.1137/1.9781611970425. URL: https://epubs.siam. org/doi/abs/10.1137/1.9781611970425.

[20] Ivo Babuška and Milo R. Dorr. "Error estimates for the combined h and p versions of the finite element method". In: Numerische Mathematik 37.2 (1981), pp. 257-277. DOI: 10 . 1007 / bf01398256.

[21] Todd A. Oliver, Krzysztof J. Fidkowski, and David L. Darmofal. "Multigrid Solution for HighOrder Discontinuous Galerkin Discretizations of the Compressible Navier-Stokes Equations". In: Computational Fluid Dynamics 2004. Springer Berlin Heidelberg, 2004, pp. 455-460. DOI: 10. 1007/3-540-31801-1_64.

[22] K. J. Fidkowski et al. "p-Multigrid solution of high-order discontinuous Galerkin discretizations of the compressible Navier-Stokes equations". In: J. Comput. Phys. 207 (2005), pp. 92-113. DoI: $10.1016 / j \cdot j$ jcp. 2005.01 .005$.

[23] K. Hillewaert et al. "Hierarchic multigrid iteration strategy for the discontinuous Galerkin solution of the steady Euler equations". In: Int. J. Meth. Numer. Fl. 51.9 (2005), pp. 1157-1176. DOI: $10.1002 / \mathrm{fld} .1135$.

[24] Hong Luo, Joseph D. Baum, and Rainald Löhner. "A p-multigrid discontinuous Galerkin method for the Euler equations on unstructured grids". In: J. Comput. Phys. 211.2 (2006), pp. 767-783. DOI: $10.1016 / j \cdot j \mathrm{jcp} .2005 .06 .019$.

[25] H. Luo, J. D. Baum, and R. Löhner. "A fast, p-Multigrid Discontinuous Galerkin Method for Compressible Flows at All Speeds”. In: AIAA 44th Aerospace Sciences Meeting. 2006. DOI: 10. 2514/6.2006-110.

[26] Brian T. Helenbrook and H. L. Atkins. "Application of p-Multigrid to Discontinuous Galerkin Formulations of the Poisson Equation”. In: AIAA Journal 44.3 (2006), pp. 566-575. DOI: 10 . 2514/1.15497.

[27] F. Bassi et al. "High-order accurate p-multigrid discontinuous Galerkin solution of the Euler equations". In: Int. J. Numer. Meth. Fluids 60 (2009), pp. 847-865. DOI: 10.1002/fld.1917.

[28] Cristian R. Nastase and Dimitri J. Mavriplis. "High-order discontinuous Galerkin methods using an hp-multigrid approach”. In: J. Comput. Phys. 213.1 (2006), pp. 330-357. DOI: 10.1016/j . jcp.2005.08.022.

[29] Klaus Stüben and Ulrich Trottenberg. "Multigrid methods: Fundamental algorithms, model problem analysis and applications". In: Lecture Notes in Mathematics. Springer Berlin Heidelberg, 1982, pp. 1-176. DOI: 10.1007/bfb0069928. 
[30] Rob Kettler. "Analysis and comparison of relaxation schemes in robust multigrid and preconditioned conjugate gradient methods". In: Lecture Notes in Mathematics. Springer Berlin Heidelberg, 1982, pp. 502-534. DOI: 10.1007/bfb0069941.

[31] Wolfgang Hackbusch. "Convergence of the Multi-Grid Iteration". In: Multi-Grid Methods and Applications. Springer Berlin Heidelberg, 1985, pp. 160-168. DOI: 10 . 1007 / 978-3-66202427-0_7.

[32] P. W. Hemker, W. Hoffmann, and M. H. van Raalte. "Two-Level Fourier Analysis of a Multigrid Approach for Discontinuous Galerkin Discretization". In: SIAM Journal on Scientific Computing 25.3 (2003), pp. 1018-1041. DOI: 10.1137/S1064827502405100.

[33] Brendan S. Mascarenhas, Brian T. Helenbrook, and Harold L. Atkins. "Coupling p-multigrid to geometric multigrid for discontinuous Galerkin formulations of the convection-diffusion equation”. In: Journal of Computational Physics 229.10 (2010), pp. 3664-3674. DOI: 10 . 1016 / j . jcp. 2010.01 .020$.

[34] Paola F. Antonietti, Marco Sarti, and Marco Verani. "Multigrid Algorithms for hp-Discontinuous Galerkin Discretizations of Elliptic Problems". In: SIAM Journal on Numerical Analysis 53.1 (2015), pp. 598-618. DOI: 10.1137/130947015.

[35] B. Cockburn and C.-W. Shu. "The Local Discontinuous Galerkin Method for Time-Dependent Convection-Diffusion Systems”. In: SIAM Journal on Numerical Analysis 35.6 (1998), pp. 24402463. DOI: $10.1137 / \mathrm{S} 0036142997316712$.

[36] Jan S. Hesthaven and Tim Warburton. Nodal Discontinuous Galerkin Methods. Springer New York, 2008. DOI: 10.1007/978-0-387-72067-8.

[37] A. Brandt. "Guide to multigrid development". In: Multigrid Methods. Ed. by W. Hackbusch and U. Trottenberg. Berlin, Heidelberg: Springer Berlin Heidelberg, 1982, pp. 220-312. ISBN: 978-3540-39544-7. 